\title{
Prognostic Value of Early Hyperglycemia in Neurocritically Ill Patients
}

\author{
Junghoon Han $^{1}$, Yun Im Lee ${ }^{2}$, Jeong-Am Ryu ${ }^{1,2}$ \\ ${ }^{1}$ Department of Neurosurgery, Samsung Medical Center, Sungkyunkwan University School of Medicine, Seoul, Korea \\ ${ }^{2}$ Department of Critical Care Medicine, Samsung Medical Center, Sungkyunkwan University School of Medicine, Seoul, Korea
}

Received: March 3, 2020

Accepted: March 24, 2020

Corresponding Author:

Jeong-Am Ryu, M.D., Ph.D.

Department of Critical Care

Medicine and Department of

Neurosurgery, Samsung Medical

Center, Sungkyunkwan University

School of Medicine, 81 Irwon-ro,

Gangnam-gu, Seoul 06351, Korea

Tel: +82-2-3410-6399

Fax: +82-2-2148-7088

E-mail: lamyud.ryu@samsung.com

\section{Objective}

To evaluate the relationship of early hyperglycemia and neurological prognosis in neurocritically ill patients.

\section{Methods}

This was a retrospective study of adult patients admitted to the neurosurgical intensive care unit (ICU) from January 2010 to July 2019. Primary outcome was neurological status at 6-month follow-up assessed with the Glasgow Outcome Scale (GOS, 1 to 5).

\section{Results}

A total of 202 patients were analyzed in this study. Of them, 70 (34.7\%) patients had early hyperglycemia ( $\geq 200 \mathrm{mg} / \mathrm{dL}$ within 48 hours after ICU admission). Brain tumor (39.6\%) and subarachnoid hemorrhage (17.8\%) were the most common reasons for ICU admission. Ninety-three (46.0\%) patients had favorable neurological outcomes (GOS of 4 or 5). Poor neurological outcome was more common in the early hyperglycemia group than in the non-hypoglycemia group $(71.4 \%$ vs. $44.7 \%, \mathrm{p}<0.001)$. In addition, 90 -day mortality rate was significantly higher for patients with early hyperglycemia than that for patients without hyperglycemia ( $17.1 \%$ vs. $8.3 \%$, $\mathrm{p}=0.011$ ). Multivariable logistic regression analysis revealed that age (adjusted odd ratio [OR]: 1.04, 95\% confidence interval (CI): 1.020-1.066), Acute Physiology and Chronic Health Evaluation II score on ICU admission (adjusted OR: 1.06, 95\% CI: 1.003-1.113), and early hyperglycemia (adjusted OR: 2.32, 95\% CI: 1.188-4.528) were significantly associated with poor neurological outcomes in neurocritically ill patients (Hosmer-Lemeshow Chi-squared $=5.43, d f=8$, $\mathrm{p}=0.711)$.

\section{Conclusions}

In this study, early hyperglycemia could be a significant predictor for neurological outcome in neurocritically ill patients.

Keywords: Hyperglycemia; Prognositc Factor; Intensive care unit; Neurosurgery 


\section{INTRODUCTION}

Hyperglycemia is common in critically ill patients. It could be associated with poor prognosis of these patients ${ }^{2,7}$. Similarly, hyperglycemia is frequently accompanied in neurocritically ill patients ${ }^{6,7)}$. Hyperglycemia is a significantly prognostic marker of poor neurological outcome of patients with ischemic stroke or intracerebral hemorrhage ${ }^{1,5,13,16)}$. However, there are limited reports of neurological prognosis according to hyperglycemia in neurocritially ill patients ${ }^{12,17}$.

Acute stress-related hyperglycemia can be develop in patients with stroke or myocardial infarction ${ }^{1,11,15)}$. Hyperglycemia may be caused by a complex interplay between counteracting regulatory hormones such as cortisol, glucagon, growth hormone, and cytokines in these patients ${ }^{17}$. Especially, early hyperglycemia is associated with mortality in patients with ST-segment elevation myocardial infarction only without diabetes mellitus $(\mathrm{DM})^{17}$. However, it is unclear whether early hyperglycemia is closely linked to prognosis in patients in neurocritically ill patients only without $\mathrm{DM}^{6}$.

Glycerin and corticosteroid are commonly used in neurocritically ill patients with severe brain edema ${ }^{12)}$. These medications can raise serum glucose levels. However, the relationship between neurological prognosis and medication-related hyperglycemia has not been reported yet. Therefore, the purpose of this study was to investigate the relationship of early hyperglycemia and neurological prognosis in neurocritically ill patients. Whether their prognosis was related to hyperglycemia itself or whether it depended on causes of hyperglycemia such as comorbidities, stress, and drugs was also investigated.

\section{METHODS}

\section{Study population and design}

This was a retrospective, single-center, observational study of adult patients admitted to the neurosurgical intensive care unit (ICU) at Samsung Medical Center from January 2010 to July 2019. This study was approved by the Institutional Review Board of Samsung Medical Center (approval number: SMC 2020-02113). The requirement for informed consent was waived due to its retrospective nature. We included adult patients admitted to the neurosurgical ICU during the study period. Of those who were hospitalized in the neurosurgical ICU for more than 7 days, we excluded patients under age 18 , those who did not have brain injury or spinal injury, those who did not have serum glucose levels within 48 hours after the neurosurgical ICU admission, and those who had insufficient medical records. Additionally, patients were excluded if they were admitted to departments other than neurosurgery.

\section{Definitions and outcomes}

We retrospectively reviewed all neurocritcally ill patients who were hospitalized in the neurosurgical ICU for more than 7 days. Serum glucose levels were measured after the neurosurgical ICU admission. Baseline glucose levels was defined as peak level within 48 hours. Minimum glucose level was determined as minimal level within 48 hours. Fluctuation of glucose level was expressed as the difference between baseline glucose level and minimal glucose level. Subjects were classified into three groups based on their baseline glucose levels (96-150 mg/dL, 151-199 mg/dL and $\geq$ $200 \mathrm{mg} / \mathrm{dL}$ ). Early hyperglycemia was defined as $\geq 200 \mathrm{mg} / \mathrm{dL}$ of baseline glucose level. The primary endpoint was poor neurological outcome at six months after the admission. This neurological status was accessed with the Glasgow Outcome Scale (GOS, 1 to 5$)^{14)}$. In this study, GOSs of 4 and 5 were classified as good neurological outcomes whereas GOSs of 1,2, and 3 were considered as poor neurological outcomes. Medical records of patients were thoroughly reviewed. Two independent intensivists (YIL and JAR) measured patients' GOSs. If the GOS did not match between these two intensivists, an agreement was reached through their discussion.

\section{Statistical analyses}

Our center has constructed the "Clinical Data Warehouse Darwin-C" designed for investigators to search and retrieve de-identified medical records from the electronic archive system. After finalizing the patient list for this study, clinical data and laboratory data were extracted from the Clinical Data Warehouse Darwin-C. All data are presented as means \pm standard deviations (SD) for continuous variables and numbers (percentages) for categorical variables. Data were compared using one-way analysis of variance with Tukey's honestly significant difference post-hoc test for continuous variables and Chi-square test or Fisher's exact test for categorical variables. Variables with P-values of less than 0.05 in univariate analyses and clinically relevant variables were subjected to a stepwise multiple logistic regression model to obtain statistically meaningful predictor variables. These clinically relevant variables were age, gender, DM, hypertension, early hyperglycemia, Acute Physiology and Chronic Health Evaluation (APACHE) II score on ICU admission, and the use of mannitol, glycerin or dexamethasone. Adequacy of the prediction model was also determined using the Hosmer-Lemeshow test. The Kaplan-Meier method was used to generate survival curves, which were compared using log-rank test. All tests were two-sided and $P$-values of 
less than 0.05 were considered statistically significant. Data were analyzed using IBM SPSS statistics version 20 (IBM, Armonk, NY, USA).

\section{RESULTS}

\section{Baseline characteristics}

A total of 202 patients were analyzed in this study. Among these patients, 70 patients (34.7\%) had early hyperglycemia. Mean age of all patients was $57.7 \pm 16.0$ years. There were 97 (48.0\%) male patients. Malignancy (56.4\%) and hypertension (45.5\%) were the most common comorbidities. Brain tumor (39.6\%) and subarachnoid hemorrhage $(17.8 \%)$ were the most common reasons for ICU admission. There was no significant difference in gender, comorbidities, reason for admission, use of mannitol, or Glasgow Coma Scale among the three groups except for age, DM, use of glycerin and dexamethasone, and APACHE II score on ICU ad- mission among baseline characteristics (Table 1).

\section{Clinical outcomes}

Among 202 neurocritically ill patients, 180 (89.1\%) patients survived until discharge from the hospital and 172 (85.1\%) patients survived until 6 months. Of these 172 survivors, 93 (46.0\%) patients had favorable neurological outcomes (GOS of 4 or 5). The entire distribution of GOS is shown in Fig. 1. Poor neurological outcome was more common in the early hyperglycemia group than that in the non-hypoglycemia group (71.4\% vs. $44.7 \%$, p < $0.001)$. In addition, in-hospital mortality was higher in the early hyperglycemia group than that in the non-hypoglycemia group ( $18.6 \%$ vs. $6.8 \%, \mathrm{p}=0.011)$. However, in patients with early hyperglycemia, poor neurological outcome was not significantly different according to the presence of DM (31.4\% vs. $40.0 \%, \mathrm{p}=0.649)$. In addition, in patients with early hyperglycemia, in-hospital mortality was not significantly different according to the presence of

Table 1. Baseline characteristics

\begin{tabular}{|c|c|c|c|c|}
\hline \multirow{2}{*}{ Variables } & \multicolumn{4}{|c|}{ Baseline glucose level (mg/dL) } \\
\hline & $96-150(n=61)$ & $151-199(\mathrm{n}=71)$ & $\geq 200(\mathrm{n}=70)$ & p-value \\
\hline Age $(y r)-$ mean $\pm S D$ & $51.0 \pm 18.0$ & $59.1 \pm 14.7^{*}$ & $62.0 \pm 13.5^{*}$ & $<0.001$ \\
\hline Gender, male — no. of patients (\%) & $27(44.3)$ & $38(53.5)$ & $32(45.7)$ & 0.508 \\
\hline \multicolumn{5}{|l|}{ Comorbidities — no. of patients (\%) } \\
\hline Malignancy & $32(52.5)$ & $38(53.5)$ & $44(62.9)$ & 0.404 \\
\hline Hypertension & $24(39.3)$ & $33(46.5)$ & $35(50.0)$ & 0.465 \\
\hline Diabetes mellitus & $3(4.9)$ & $8(11.3)$ & $32(45.7)$ & 0.001 \\
\hline Current smoker & $10(16.4)$ & $11(15.5)$ & $10(14.3)$ & 0.945 \\
\hline Ischemic heart disease & $2(3.3)$ & $5(7.0)$ & $7(10.0)$ & 0.322 \\
\hline Chronic kidney disease & $1(1.6)$ & $7(9.9)$ & $5(7.1)$ & 0.130 \\
\hline Cause of ICU admission — no. of patients (\%) & & & & 0.091 \\
\hline Brain tumor & $24(39.3)$ & $27(38.0)$ & $29(41.4)$ & \\
\hline Subarachnoid hemorrhage & $11(18.0)$ & $16(22.5)$ & $9(12.9)$ & \\
\hline Intracranial hemorrhage & $10(16.4)$ & $15(21.1)$ & $7(10.0)$ & \\
\hline Traumatic brain injury & $4(6.6)$ & $7(9.9)$ & $15(21.4)$ & \\
\hline Cerebral infarction & $1(1.6)$ & $2(2.8)$ & $3(4.3)$ & \\
\hline Central nerve system infection & $2(3.3)$ & $0(0)$ & $3(4.3)$ & \\
\hline Other & $9(14.8)$ & $4(5.6)$ & $4(5.7)$ & \\
\hline \multicolumn{5}{|l|}{ Use of drugs — no. of patients (\%) } \\
\hline Mannitol & $49(80.3)$ & $63(88.7)$ & $51(72.9)$ & 0.058 \\
\hline Glycerin & $14(23.0)$ & $24(33.8)$ & $31(44.3)$ & 0.037 \\
\hline Dexamethasone & $8(13.1)$ & $28(39.4)$ & $24(34.3)$ & 0.003 \\
\hline Glycerin and dexamethasone & $2(3.3)$ & $9(12.7)$ & $13(18.6)$ & 0.025 \\
\hline GCS on ICU admission - mean \pm SD & $7.7 \pm 4.6$ & $7.7 \pm 4.2$ & $6.6 \pm 4.1$ & 0.136 \\
\hline APACHE II score on ICU admission - mean \pm SD & $19.6 \pm 6.9^{*}$ & $19.4 \pm 6.2^{*}$ & $22.3 \pm 6.0$ & 0.015 \\
\hline
\end{tabular}

Data are expressed as mean \pm standard deviation (SD) or frequencies (\%). Statistical significances of continuous variables among groups were tested with oneway analysis of variance. The same letters indicate non-significant differences between groups base on the Tukey's honestly significant difference post-hoc test. APACHE: Acute Physiology and Chronic Health Evaluation; GCS: Glasgow Coma Scale; ICU: intensive care unit.

*The same letters indicate non-significant differences between groups base on the Tukey's honestly significant difference post-hoc test. 
$\operatorname{DM}(4.3 \%$ vs. $14.3 \%, \mathrm{p}=0.069$, Table 2$)$.

Minimum glucose level was higher in patients with poor neurological outcome than those with good neurological outcome $(154.7 \pm 46.0 \mathrm{mg} / \mathrm{dL}$ vs. $139.5 \pm 30.6 \mathrm{mg} / \mathrm{dL}, \mathrm{p}=0.006)$. Fluctuation of glucose level did not show significant difference between patients with poor neurological outcome and those with good neurological outcome $(56.1 \pm 70.8 \mathrm{mg} / \mathrm{dL}$ vs. $39.8 \pm 46.3 \mathrm{mg} / \mathrm{dL}$, $\mathrm{p}=0.052$ ).

Multivariable logistic regression analysis revealed that age (adjusted odd ratio [OR]: 1.04, 95\% confidence interval (CI):

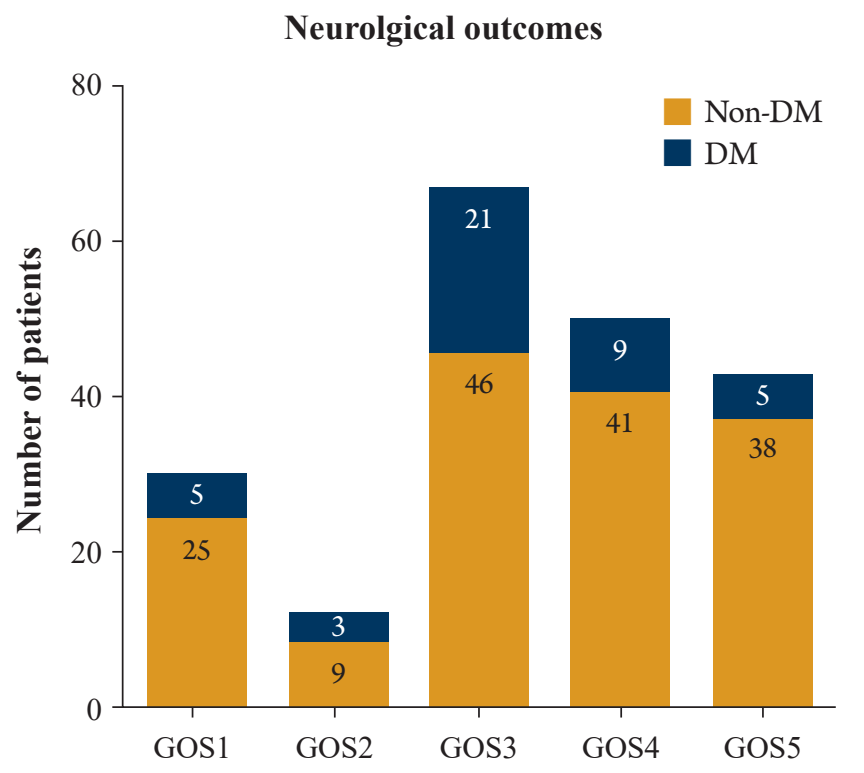

Glasgow outcome scale

Fig. 1. Entire distribution of Glasgow Outcome Scale (GOS) after 6 months according to diabetes mellitus (DM).
1.020-1.066), APACHE II score on ICU admission (adjusted OR: 1.06, 95\% CI: 1.003-1.113), and early hyperglycemia (adjusted OR: 2.32 , 95\% CI: 1.188-4.528) were significantly associated with poor neurological outcomes in neurocritically ill patients (Hosmer-Lemeshow Chi-squared $=5.43, d f=8, \mathrm{p}=0.711$, Table 3 ). In addition, the 90 -day mortality rate was significantly lower in patients without early hyperglycemia compared to those with early hyperglycemia ( $8.3 \%$ vs $17.1 \%$, log-rank test, $\mathrm{p}=0.011$, Fig. 2).

\section{DISCUSSION}

In this study, we investigated the relationship of early hyperglycemia and neurological prognosis in neurocritically ill patients. This study had the following major findings. First, poor neurological outcome was more common in the early hyperglycemia group than that in the non-hypoglycemia group. In-hospital mortality

Table 3. Multivariable logistic regression for the relationship of clinically relevant variables associated with poor neurological outcome

\begin{tabular}{|c|c|c|c|}
\hline Variables & $B$ & Adjusted OR (95\% CI) & $\mathrm{p}$-value \\
\hline Age & 0.042 & $1.04(1.020-1.066)$ & $<0.001$ \\
\hline $\begin{array}{l}\text { APACHE II score on ICU } \\
\text { admission }\end{array}$ & 0.055 & $1.06(1.003-1.113)$ & 0.037 \\
\hline Hyperglycemia & 0.841 & $2.32(1.188-4.528)$ & 0.014 \\
\hline
\end{tabular}

Variables with p-values under 0.05 in univariate analyses and clinically relevant variables were subjected to a stepwise multiple logistic regression model to obtain statistically meaningful predictor variables. They were age, gender, diabetes mellitus, hypertension, hyperglycemia, Acute Physiology and Chronic Health Evaluation (APACHE) II score on intensive care unit (ICU) admission, and the use of mannitol, glycerin or dexamethasone. CI: confidence interval; OR: odd ratio.

Table 2. Clinical outcomes

\begin{tabular}{|c|c|c|c|c|}
\hline \multirow{2}{*}{ Variables } & \multicolumn{4}{|c|}{ Baseline glucose level (mg/dL) } \\
\hline & $96-150(n=61)$ & $151-199(n=71)$ & $\geq 200(n=70)$ & $\mathrm{p}$-value \\
\hline Poor neurological outcome - no. of patients (\%) & $25(41.0)$ & $34(47.9)$ & $50(71.4)$ & 0.001 \\
\hline DM patients & $1(1.6)$ & $6(8.5)$ & $22(31.4)$ & \\
\hline Non-DM patients & $24(39.3)$ & $28(39.4)$ & $28(40.0)$ & \\
\hline ICU mortality — no. of patients (\%) & $1(1.6)$ & $6(8.5)$ & $9(12.9)$ & 0.059 \\
\hline DM patients & $0(0)$ & $0(0)$ & $2(2.9)$ & \\
\hline Non-DM patients & $1(1.6)$ & $6(8.5)$ & $7(10.0)$ & \\
\hline Hospital mortality — no. of patients (\%) & $2(3.3)$ & $7(9.9)$ & $13(18.6)$ & 0.019 \\
\hline DM patients & $0(0)$ & $0(0)$ & $3(4.3)$ & \\
\hline Non-DM patients & $2(3.3)$ & $7(9.9)$ & $10(14.3)$ & \\
\hline Length of stay in ICU (days) - mean \pm SD & $14.0 \pm 16.2$ & $10.5 \pm 6.3$ & $14.8 \pm 11.3$ & 0.068 \\
\hline Length of stay in hospital (days) - mean \pm SD & $48.6 \pm 33.9$ & $58.6 \pm 138.7$ & $66.1 \pm 86.9$ & 0.597 \\
\hline
\end{tabular}

DM: diabetes mellitus; ICU: intensive care unit; SD: standard deviation. 


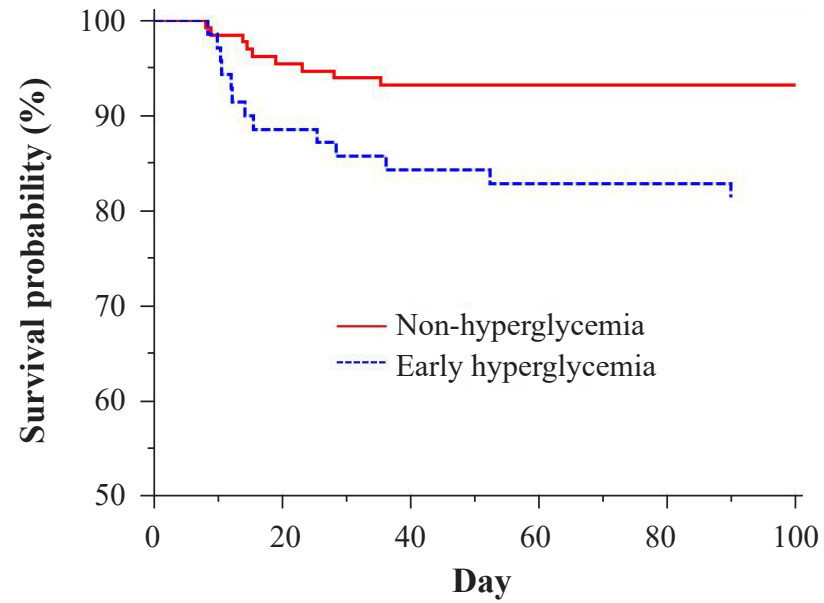

Fig. 2. Kaplan-Meier 90-day survival analysis comparing early hyperglycemia and nonhyperglycemia groups of neurocritically ill patients. Red solid line, non-hyperglycemia; blue dotted line, early hyperglycemia. $\mathrm{p}=0.011$, based on log-rank tests.

was also higher in the early hyperglycemia group than that in the non-hypoglycemia group. Second, in patients with early hyperglycemia, clinical prognoses were similar between DM group and non-DM group. Third, multivariable logistic regression analysis revealed that age, APACHE II score on ICU admission, and early hyperglycemia were significantly associated with poor neurological outcomes in neurocritically ill patients. Fourth, early hyperglycemia itself could be a significant predictor for poor neurological outcome in neurocritically ill patients regardless of comorbidities or drugs.

Hyperglycemia is associated with poor prognosis in various ICU patients $^{2,3,8,10)}$. Hyperglycemia is a predictor associated with poor neurological prognosis in patients with neurological diseases such as ischemic stroke, intracerebral hemorrhage, and aneurysmal subarachnoid hemorrhage ${ }^{5,9,13,16)}$. Indeed, hyperglycemia itself could be harmful to the brain ${ }^{7}$. Complex factors such as free radical formation and oxidative injury, activation of N-methyl-D-aspartate receptors, raised intracellular calcium, triggering of inflammatory and apoptotic pathways, and alterations in lactate metabolism are associated with primary toxic-ischemic injury and secondary ${ }^{1,4,77}$. In addition, ischemic penumbra could be injured due to direct neurotoxic effect arising from hyperglycemia ${ }^{1}$.

In critically ill patients, hyperglycemia could be caused by various factors ${ }^{2,7)}$. Especially, stress-related hyperglycemia mainly results from the release of stress hormones caused by acute illness ${ }^{6}$. In addition, stress-related hyperglycemia might be associated with the extent and severity of ischemic damage in patients with stroke or ST-segment elevation myocardial infarction ${ }^{1,17}$. Therefore, stress-related hyperglycemia might be associated with the progno- sis of critically ill patients. A few studies have shown that the relationship between hyperglycemia and poor prognosis is unclear in DM patients ${ }^{17}$. Although stress-related hyperglycemia could be important to predict the prognosis of critically ill patients, it might be confused with DM-related hyperglycemia. In this study, regardless of DM or use of drugs, early hyperglycemia itself was associated with poor neurological outcome in neurocritically ill patients. This relationship might be due to a neurotoxic effect of hyperglycemia itself in patients with brain injuries.

In this study, early hyperglycemia occurred more frequently in patients who used glycerine or dexamethasone than in those who did not. Corticosteroid and multiple osmotic agents might be used in patients with severe brain damages. Therefore, hyperglycemia might arise from these medications in more sick patients. However, in multivariable variable analysis, the use of glycerine or dexamethasone was not associated with poor neurological outcomes. Neurological prognosis was more associated with early hyperglycemia itself compared to the use of glycerine or dexamethasone in this study. Therefore, it is necessary to consider drug-related hyperglycemia when treating neurocritically ill patients.

In this study, early hyperglycemia associated with poor neurological outcome in neurocritically ill patients. Especially, early hyperglycemia itself could elevate risk of poor neurological outcome in these patients. Therefore, aggressively hyperglycemic control could be important to protect their brain from hyperglycemia inducing brain damage in patients with early hyperglycemia. In addition, management of these patients needs to be aware of drugs that are at risk for inducing hyperglycemia, such as glycerin and dexamethasone.

This study has several limitations. First, it was a retrospective review of medical records. Second, administration of corticosteroid or multiple osmotic agents was determined by a neurointensivist or a neurosurgeon. It was not protocol-based. Therefore, the non-randomized nature of registry data might have resulted in selection bias. Third, in a few patients, causes of hyperglycemia were difficult to distinguish between hyperglycemia caused by stress or DM if DM was not diagnosed on admission. Finally, our study had limited statistical power due to its small sample size. Although this study provides valuable insight, prospective large-scale studies are needed to evaluate the relationship of early hyperglycemia and neurological prognosis in neurocritically ill patients to obtain evidence-based conclusions.

\section{CONCLUSION}

In this study, poor neurological outcome in neurocritcally ill patients was associated with early hyperglycemia. In addition, DM, 
the use of corticosteroid, or the use of multiple osmotic agents was not associated with neurological prognosis. Therefore, early hyperglycemia itself could be a significant predictor associated with neurological outcome in neurocritically ill patients.

\section{NOTES}

\section{Conflict of interest}

We declare that we have no conflict of interest.

\section{Informed consent}

Informed consent was obtained from each participant included in this study.

\section{Acknowledgements}

We would like to thank Hye Jung Kim, nursing director of the neurosurgical intensive care unit, for providing excellent advice and engaging in fruitful discussions. We would also like to thank all nurses of the neurosurgical intensive care unit at Samsung Medical Center.

\section{REFERENCES}

1. Capes SE, Hunt D, Malmberg K, Pathak P, Gerstein HC. Stress hyperglycemia and prognosis of stroke in nondiabetic and diabetic patients: a systematic overview. Stroke 2001;32:24262432.

2. Finfer S, Chittock DR, Su SY, Blair D, Foster D, Dhingra V, et al. Intensive versus conventional glucose control in critically ill patients. N Engl J Med 2009;360:1283-1297.

3. Gale SC, Sicoutris C, Reilly PM, Schwab CW, Gracias VH. Poor glycemic control is associated with increased mortality in critically ill trauma patients. Am Surg 2007;73:454-460.

4. Godoy DA, Di Napoli M, Rabinstein AA. Treating hyperglycemia in neurocritical patients: benefits and perils. Neurocrit Care 2010; $13: 425-438$.

5. Gofir A, Mulyono B, Sutarni S. Hyperglycemia as a prognosis predictor of length of stay and functional outcomes in patients with acute ischemic stroke. Int J Neurosci 2017;127:923-929.

6. Guo YJ, Zhou Y, Zhang SY, Wei Q, Huang Y, Xia WQ, et al. Optimal target range for blood glucose in hyperglycaemic patients in a neurocritical care unit. Diab Vasc Dis Res 2014;11:352358.

7. Kramer AH, Roberts DJ, Zygun DA. Optimal glycemic control in neurocritical care patients: a systematic review and meta-analysis. Crit Care 2012; 16:R203.

8. Krinsley JS. Association between hyperglycemia and increased hospital mortality in a heterogeneous population of critically ill patients. Mayo Clin Proc 2003;78:1471-1478.

9. Kruyt ND, Biessels GJ, DeVries JH, Luitse MJ, Vermeulen M, Rinkel GJ, et al. Hyperglycemia in aneurysmal subarachnoid hemorrhage: a potentially modifiable risk factor for poor outcome. J Cereb Blood Flow Metab 2010;30:1577-1587.

10. Malmberg K, Ryden L, Wedel H, Birkeland K, Bootsma A, Dickstein $\mathrm{K}$, et al. Intense metabolic control by means of insulin in patients with diabetes mellitus and acute myocardial infarction (DIGAMI 2): effects on mortality and morbidity. Eur Heart J 2005;26:650-661.

11. Melamed E. Reactive hyperglycaemia in patients with acute stroke. J Neurol Sci 1976;29:267-275.

12. Ryu JA, Jung W, Jung YJ, Kwon DY, Kang K, Choi H, et al. Early prediction of neurological outcome after barbiturate coma therapy in patients undergoing brain tumor surgery. PLoS One 2019;14:e0215280.

13. Saxena A, Anderson CS, Wang X, Sato S, Arima H, Chan E, et al. Prognostic Significance of Hyperglycemia in Acute Intracerebral Hemorrhage: The INTERACT2 Study. Stroke 2016; 47:682-688.

14. Sekhon MS, Griesdale DE, Robba C, McGlashan N, Needham $\mathrm{E}$, Walland $\mathrm{K}$, et al. Optic nerve sheath diameter on computed tomography is correlated with simultaneously measured intracranial pressure in patients with severe traumatic brain injury. Intensive Care Med 2014;40:1267-1274.

15. Sewdarsen M, Jialal I, Vythilingum S, Govender G, Rajput MC. Stress hyperglycaemia is a predictor of abnormal glucose tolerance in Indian patients with acute myocardial infarction. Diabetes Res 1987;6:47-49.

16. Tsivgoulis G, Katsanos AH, Mavridis D, Lambadiari V, Roffe C, Macleod MJ, et al. Association of Baseline Hyperglycemia With Outcomes of Patients With and Without Diabetes With Acute Ischemic Stroke Treated With Intravenous Thrombolysis: A Propensity Score-Matched Analysis From the SITS-ISTR Registry. Diabetes 2019;68:1861-1869.

17. Yang JH, Song PS, Song YB, Hahn JY, Choi SH, Choi JH, et al. Prognostic value of admission blood glucose level in patients with and without diabetes mellitus who sustain ST segment elevation myocardial infarction complicated by cardiogenic shock. Crit Care 2013;17:R218. 